\title{
Collagenous Fibroma at the Surgical Site after Thyroidectomy for Papillary Thyroid Carcinoma
}

\author{
Ji Min Yun ${ }^{1}$, Sunhee Chang ${ }^{2}$, and Yoon Kyoung So ${ }^{1}$ \\ ${ }^{1}$ Departments of Otorhinolaryngology-Head and Neck Surgery, ${ }^{2}$ Pathology, Ilsan Paik Hospital, Inje University College of Medicine, \\ Goyang, Korea
}

갑상선 유두암 수술 부위에 발생한 교원질성 섬유종 1예

윤지민 ${ }^{1} \cdot$ 장선희 ${ }^{2} \cdot$ 소윤경

인제대학교 의과대학 일산백병원 이비인후과학교실, ${ }^{1}$ 병리학교실 ${ }^{2}$

Received October 5, 2020

Revised November 29, 2020

Accepted December 18, 2020

Address for correspondence

Yoon Kyoung So, MD, PhD

Department of Otorhinolaryngology-

Head and Neck Surgery,

Ilsan Paik Hospital,

Inje University College of Medicine,

170 Juhwa-ro, Ilsanseo-gu,

Goyang 10380, Korea

Tel $+82-31-910-7250$

Fax $+82-31-910-7518$

E-mail hn0131@paik.ac.kr
Collagenous fibroma (CF) is a rare benign tumor that occurs at various anatomical sites including the head and neck. CFs are often omitted from differential diagnosis before surgery. We describe a case of CF in a 54-year-old male who presented with a growing neck mass of 1 -year duration at the site of previous right hemithyroidectomy for papillary thyroid carcinoma (PTC). Although radiologic findings demonstrated a benign tumor, surgical excision was carried out per patient's preference and to rule out recurrent thyroid cancer. The mass was severely adherent to the surrounding muscles, but was resected completely without any injury to the adjacent structures. Histological studies revealed CF. The postoperative course was uneventful without vocal fold paralysis. CFs can be a diagnostic challenge in preoperative evaluation. Conservative excision was sufficient in most cases without recurrence. Clinicians should be aware of CFs in diagnosis, especially in postoperative site.

Korean J Otorhinolaryngol-Head Neck Surg 2021;64(12):932-6

Keywords Collagenous fibroma; Desmoplastic fibroblastoma; Papillary thyroid carcinoma; Recurrence; Thyroidectomy.

\section{Introduction}

Collagenous fibroma (CF) is a rare, benign fibroblastic soft tissue tumor that classically presents as a non-tender, firm, slow-growing mass. This type of tumor was first described in 1995 by Evans, ${ }^{1)}$ who coined the term 'desmoplastic fibroblastoma,' and was later renamed 'CF' by Nielsen, et al. ${ }^{2)}$ The largest series of $\mathrm{CF}$ with 63 cases was published by Miettinen and Fetsch, ${ }^{3)}$ who elucidated the clinicopathological features of the lesion. CFs predominantly occur within the subcutaneous tissue, but skeletal muscle involvement is common.

This is an Open Access article distributed under the terms of the Creative Commons Attribution Non-Commercial License (https://creativecommons.org/licenses/by-nc/4.0) which permits unrestricted non-commercial use, distribution, and reproduction in any medium, provided the original work is properly cited.
They can occur at various sites, including arm, shoulder, back, extremities, head, and neck. ${ }^{3-10)} \mathrm{CFs}$ are often omitted from differential diagnosis before surgery. In cases in which the mass develops at the site of a previous surgical wound, it can be mistaken for a recurrent tumor.

We report a unique case of $\mathrm{CF}$ that occurred at a previous surgical site after thyroidectomy for papillary thyroid carcinoma (PTC). Despite the rarity of CF, identification of this tumor is of diagnostic and therapeutic importance because it can be misdiagnosed as recurrent PTC, and surgery can risk damaging surrounding structures, including the recurrent laryngeal nerve (RLN). 


\section{Case}

A 54-year-old male with a history of right-sided thyroid cancer presented with a mass that had been growing at a previous surgical site for one year. He had undergone right thyroid lobectomy for PTC through a cervical approach seven years prior at another hospital. At that time, lymph node dissection had not been performed. He had been taking levothyroxine $0.075 \mathrm{mg}$ daily since the surgery. The patient detected a painless mass on his right anterior neck one year before presentation (six years after thyroidectomy). Ultrasonography (US) performed at the other hospital revealed a $2.7 \times 1.8 \times 3.0-\mathrm{cm}$ sized, hypoechoic mass at the thyroidectomy bed, and USguided fine needle aspiration biopsy (FNAB) yielded fibromuscular tissue with no tumor cells. On follow-up US one year later, the mass increased in size to $3.2 \times 2.2 \times 3.8 \mathrm{~cm}$, and the patient was referred to our hospital for further evaluation.

Physical examination revealed a 3.5-cm-sized, firm, nontender mass around the previous thyroidectomy site in the right lower anterior neck. Vocal fold mobility was normal bilaterally, and there was no narrowing of the airway. Computed tomog- raphy $(\mathrm{CT})$ scan demonstrated an approximately $4 \mathrm{~cm}$, nonenhancing mass in the right thyroidectomy bed (Fig. 1). Enlarged cervical lymph nodes were not seen on CT. US showed a well-circumscribed, isoechoic mass at the right thyroidectomy bed in contact with the trachea (Fig. 1). US-guided FNAB was performed, but the needle barely penetrated the mass. The result of FNAB was non-diagnostic with no cellular component. Considering the radiological hypothesis of a benign soft tissue tumor and patient's preference of surgical removal, authors scheduled a surgical excision rather than further radiologic or histologic evaluation, but we could not completely ruled out recurrent thyroid cancer preoperatively because of it's location and rapid growing entity. With the patient under general anesthesia, the mass was excised with intraoperative monitoring of the RLN (Fig. 2). Considering potential adhesion to the previous thyroidectomy wound and the large size of the mass, we approached the mass from the lateral border of the right strap muscles. Grossly, the mass was firm, wellcircumscribed, 3.5-cm-sized, and severely adherent to the adjacent sternocleidomastoid muscle (SCM) and strap muscles. The mass contacted the trachea but was easily dissected from
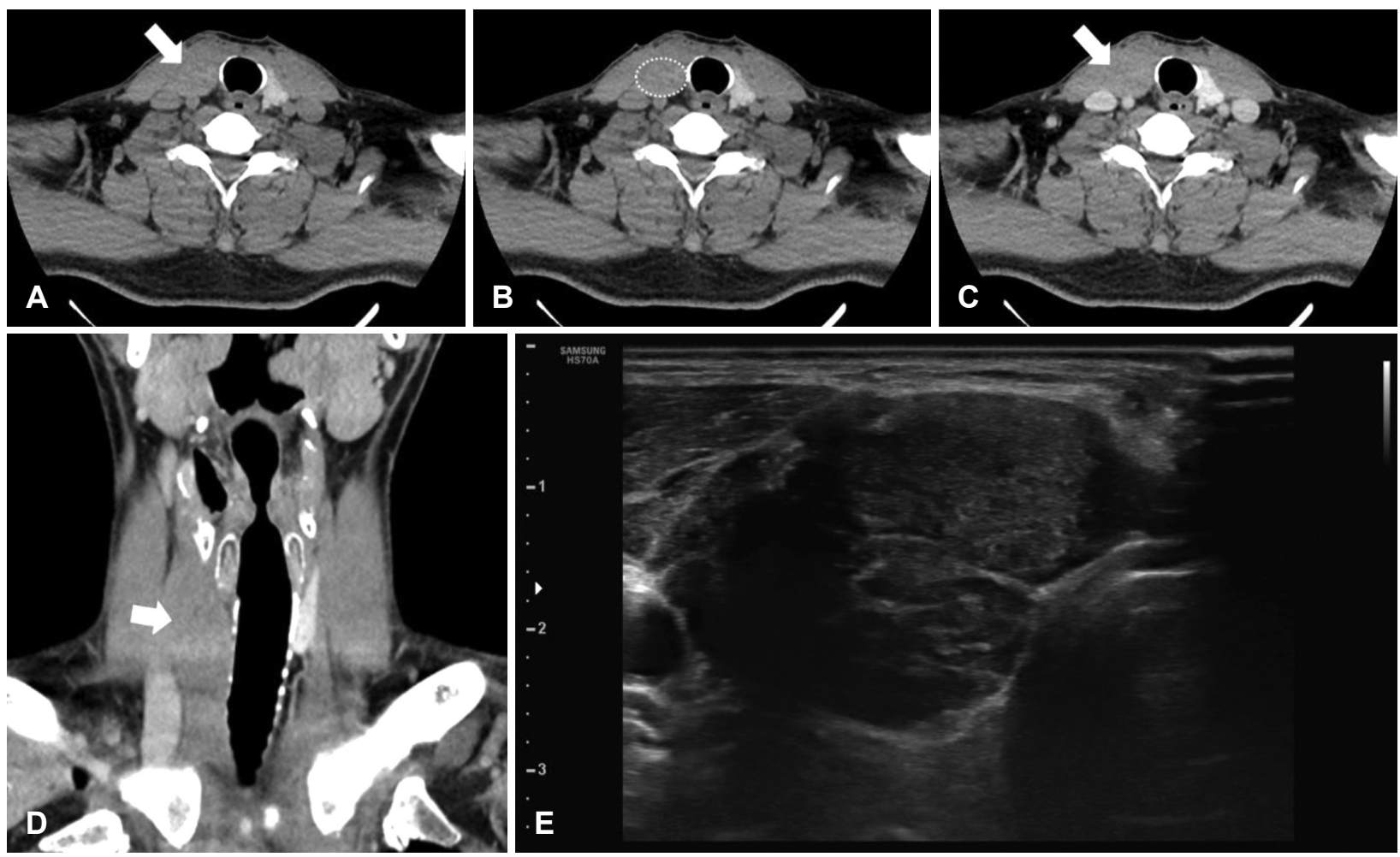

Fig. 1. Radiographic images of the tumor. On CT scan, an approximately $4 \mathrm{~cm}$, ill-demarcated mass was observed at the right thyroidectomy bed (A-D). On an axial pre-enhanced CT scan, the mass showed the same density as the surrounding muscles (arrow, A). The outline of the mass is indicated with a dotted line (B). On contrast-enhanced CT scans, the mass was not enhanced (arrows; C, D). Rather, US (E) showed a well-circumscribed, isoechoic mass in contact with the trachea, although US-guided fine needle aspiration had no yield. US: ultrasonography. 

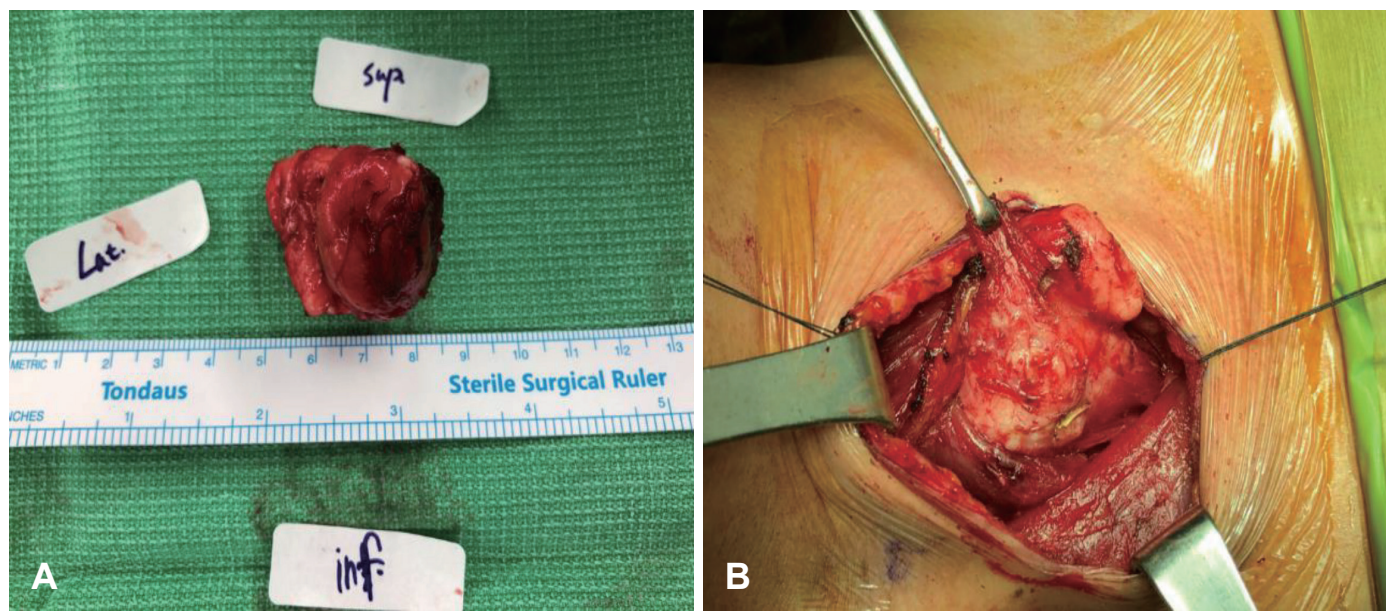

Fig. 2. Gross findings of the tumor. Grossly, the mass was firm, well-circumscribed, and $3.5 \mathrm{~cm}$ in size (A). The mass was located in the right thyroidectomy bed and severely adherent to adjacent sternocleidomastoid and strap muscles. It contacted the trachea but was easily dissected from it (B).
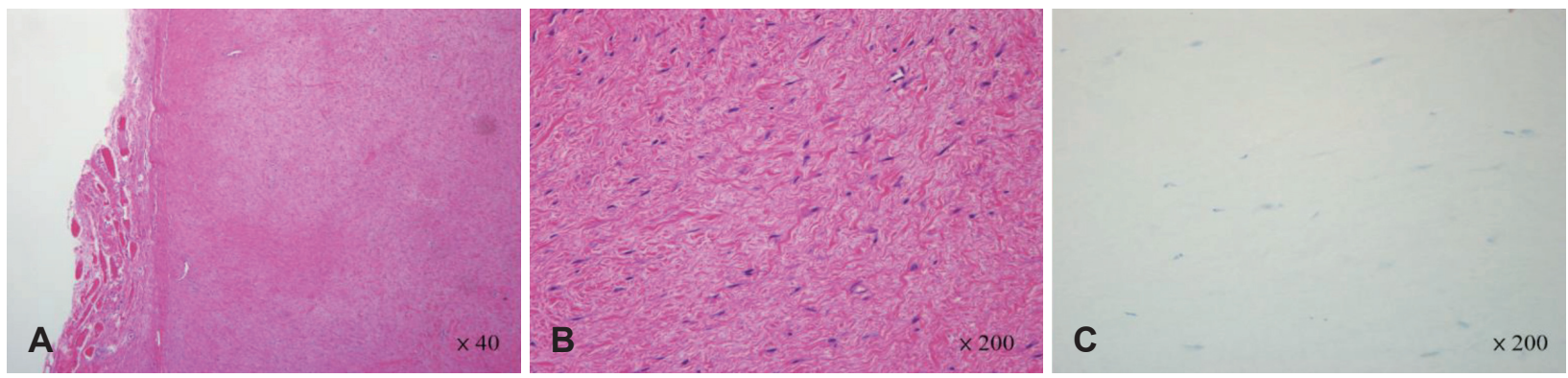

Fig. 3. Microscopic findings of the tumor. The tumor was well-circumscribed and hypocellular (H\&E stain, $\times 40)(A)$. The tumor showed a patternless distribution of bland spindle- and stellate-shaped cells in a prominent collagenous-to-myxoid matrix $(H \& E, \times 200)(B)$. Neither mitotic figure nor necrosis was found. The tumor cells did not show aberrant nuclear expression on beta-catenin immunochemical stain (Beta-catenin, ×200) (C). H\&E: hematoxylin and eosin.

it. Although the RLN was not identified during the operation, no sign of nerve injury was recorded with nerve monitoring. On pathologic examination, the mass was well circumscribed and hypocellular (Fig. 3). Within the tumor, a patternless distribution of bland spindle- or stellate-shaped cells was noted in a prominent collagenous-to-myxoid matrix. No mitotic figure or necrosis was found. The tumor cells did not show aberrant nuclear beta-catenin expression. The patient's postoperative course was uneventful without complications, and vocal fold mobility was intact bilaterally.

\section{Discussion}

$\mathrm{CF}^{2)}$ previously known as desmoplastic fibroblastoma, ${ }^{1)}$ is a rare benign fibroblastic/myofibroblastic neoplasm that most frequently involves the extremities and upper back. ${ }^{3)}$ Even rarer, it develops in the head and neck area, including the parotid, neck, and palate areas. ${ }^{4,6-10)} \mathrm{CF}$ was reported to have a male predominance $(51: 12)$ and to have a peak incidence in the fifth and sixth decades of life. ${ }^{3)}$ Clinically, CF presents as a solitary, firm, well-circumscribed, painless and slow-growing mass of long duration. In majority of cases, no inciting event was clinically mentioned and no specific cause of a reactive fibrous proliferation was microscopically identified, suggesting that CFs represent neoplasms rather than reactive condition. ${ }^{1,2)}$ However, a history of antecedent trauma was previously noted in a few cases. ${ }^{3,6)}$ Since no case of CFs presenting at a postoperative site have been reported before, this case may indicate possibility of reactive condition involvement in development of CFs.

We report a unique case of CF that developed at a previous thyroidectomy site. CF can be a diagnostic challenge. The clinical features of the tumor in the present case, which occurred in the thyroidectomy bed from previous thyroid cancer and increased in size over 1 year, could have misled to diagnosis of recurrent thyroid cancer. Although both US and CT scans demonstrated benign tumor, recurrent thyroid cancer was not ruled out considering the indolent nature of differentiated thy- 
roid cancers. Furthermore, the result of FNAB was non-diagnostic. Given the hypocellularity of the tumor, the FNAB result was low yield, and a core biopsy or incisional biopsy could have been more helpful. CF can also be a surgical challenge. Because the mass occupied the previous thyroidectomy site, revision surgery at this site risked damaging various vital structures, such as the RLN, trachea, esophagus, and surrounding neck muscles. According to recent reports, most CFs are located in the subcutaneous fat layer, but a muscular location is not rare $(27 \%)$. Despite good demarcation, infiltration into fat or skeletal muscle was reported in most cases $(78 \%)$. ${ }^{3)}$ Also, there have been reports of neurologic deficits in cases of large $\mathrm{CF}^{6,11-13)}$ Those can be worrisome characteristics for excision, especially in cases requiring revision surgery. In this case, the huge mass was severely adherent to the strap muscles and SCM. There was no adhesion to or invasion of the RLN, trachea, or esophagus. Postoperatively, there was no complication related to damage of surrounding structures.

CF is usually diagnosed postoperatively. Grossly, the tumor is well encapsulated and has a firm, homogeneous consistency. Microscopically, it is typically hypocellular with a highly collagenous or myxocollagenous matrix. ${ }^{5)}$ Uniform, spindleto-stellate-shaped myofibroblasts scatter in fibromyxoid stroma, and mitotic activity is extremely low. All these pathologic features were observed in this case. The most important differential diagnosis for CF is desmoid-type fibromatosis (DTF), also called desmoid tumor. Microscopically, DTF is also characterized by spindle cells of uniform appearance with abundant collagen, but it is distinguished from CF by increased cellularity, poor circumscription, and an infiltrating border. ${ }^{14)}$ While CF has a benign nature and never recurs after simple excision, DTF is generally locally aggressive and recurs frequently. In addition to aggressive pathological findings in DTF, immunohistochemical staining for beta-catenin can help the differential diagnosis. Nuclear beta-catenin is reported to be positive in most, if not all, cases of DTF. ${ }^{14,15)}$ In this case, the tumor was hypocellular, and nuclear beta-catenin was negative. Other hypercellular fibrous tumors for differential diagnosis include fibroma of tendon sheath, calcifying fibrous pseudotumor, nodular fasciitis, and low-grade fibromyxoid sarcoma.

Another point of interest is the tissue of origin in this case. Since the tumor was adherent to the adjacent strap muscles and occupied thyroidectomy bed, we believe that in this case the tissue of origin could have been the strap muscles probably irritated by previous surgery. Previously reported case of $\mathrm{CF}$ involving thyroid gland suggested the prevertebral fascia surrounding the thyroid gland or carotid sheath as the possible origin. $^{10)}$

Surgery has been the historical standard of treatment for $\mathrm{CFs}$, and tumor recurrence after excision has not been reported. ${ }^{3)}$ Considering the benign nature of this tumor and lack of recurrence, conservative excision is recommended to minimize potential morbidity.

We described the unusual case of CF that developed at the surgical site of PTC. Because a soft tissue mass at the previous surgical site can be misdiagnosed as a recurrent tumor and CFs are difficult to be diagnosed with preoperative workups, it is clearly a diagnostic challenge. It may also pose a therapeutic challenge because it risks the injury of adjacent structures at the revision surgery of the thyroidectomy site. Despite these diagnostic and therapeutic difficulties, recurrence has not been reported after complete resection.

\section{Acknowledgments}

None.

\section{Author Contribution}

Conceptualization: Ji Min Yun, Yoon Kyoung So. Data curation: all authors. Formal analysis: all authors. Funding acquisition: Ji Min Yun, Yoon Kyoung So. Investigation: Ji Min Yun. Methodology: Ji Min Yun, Yoon Kyoung So. Project administration: Ji Min Yun. Resources: Ji Min Yun. Software: Ji Min Yun. Supervision: Yoon Kyoung So. Validation: Yoon Kyoung So. Visualization: Ji Min Yun, Sunhee Chang. Writing — original draft: Ji Min Yun. Writing - review \& editing: Yoon Kyoung So.

\section{ORCID}

Yoon Kyoung So https://orcid.org/0000-0001-9990-4293

\section{REFERENCES}

1) Evans HL. Desmoplastic fibroblastoma. A report of seven cases. Am J Surg Pathol 1995;19(9):1077-81.

2) Nielsen GP, O'Connell JX, Dickersin GR, Rosenberg AE. Collagenous fibroma (desmoplastic fibroblastoma): A report of seven cases. Mod Pathol 1996;9(7):781-5.

3) Miettinen M, Fetsch JF. Collagenous fibroma (desmoplastic fibroblastoma): A clinicopathologic analysis of 63 cases of a distinctive soft tissue lesion with stellate-shaped fibroblasts. Hum Pathol 1998;29(7):676-82.

4) Dagli M, Eryilmaz A, Acar A, Kulacoglu S, Akmansu H. Collagenous fibroma (desmoplastic fibroblastoma). Yonsei Med J 2004;45(5): 941-3.

5) Farlow JL, McHugh JB, Spector ME. A slow-growing fibrous parapharyngeal mass. JAMA Otolaryngol Head Neck Surg 2018; 144(10):944-5.

6) Fong F, Odell E, Simo R. Collagenous fibroma (desmoplastic fibroblastoma) of the neck presenting with neurological symptoms. Head Neck Pathol 2009;3(1):47-50.

7) Ide F, Shimoyama T, Horie N, Tanaka H. Collagenous fibroma (desmoplastic fibroblastoma) presenting as a parotid mass. J Oral Pathol Med 1999;28(10):465-8.

8) Mesquita RA, Okuda E, Jorge WA, de Araújo VC. Collagenous 
fibroma (desmoplastic fibroblastoma) of the palate: A case report. Oral Surg Oral Med Oral Pathol Oral Radiol Endod 2001;91(1):80-4.

9) Shimoyama T, Horie N, Ide F. Collagenous fibroma (desmoplastic fibroblastoma): A new case originating in the palate. Dentomaxillofac Radiol 2005;34(2):117-9.

10) Wilson C, Summerall J, Lubin J, Mesko TW. Collagenous fibroma (desmoplastic fibroblastoma): A unique presentation as a goiter in an 88-year-old man. Ann Diagn Pathol 2000;4(3):165-9.

11) Hao S, Cherian R, Templeton KJ, Topalovski M, McGregor DH. Collagenous fibroma (desmoplastic fibroblastoma) of pectoralis minor muscle. Orthopedics 2003;26(12):1227-8.

12) Hasegawa T, Shimoda T, Hirohashi S, Hizawa $K$, Sano T. Collagenous fibroma (desmoplastic fibroblastoma): Report of four cases and review of the literature. Arch Pathol Lab Med 1998; 122(5):455-60.

13) Osipov V, Carrera GF. Collagenous fibroma (desmoplastic fibroblastoma) with vertebral body erosion. Sarcoma 2009;2009: 682687.

14) Martínez Trufero J, Pajares Bernad I, Torres Ramón I, Hernando Cubero J, Pazo Cid R. Desmoid-type fibromatosis: Who, when, and how to treat. Curr Treat Options Oncol 2017;18(5):29.

15) Koike H, Nishida $Y$, Kohno K, Shimoyama Y, Motoi T, Hamada S, et al. Is immunohistochemical staining for $\beta$-catenin the definitive pathological diagnostic tool for desmoid-type fibromatosis? A multi-institutional study. Hum Pathol 2019;84:155-63. 